\title{
Foraging with Anne Treisman: Features versus conjunctions, patch leaving and memory for foraged locations
}

\author{
Árni Kristjánsson ${ }^{1,2} \cdot$ Andri S. Björnsson ${ }^{1} \cdot$ Tómas Kristjánsson ${ }^{1}$
}

Published online: 2 January 2020

(C) The Psychonomic Society, Inc. 2020

\begin{abstract}
Foraging tasks are increasingly used to investigate human visual attention as they may provide a more dynamic and multifaceted picture of attentional orienting than more traditionally used visual search tasks. A common way of assessing foraging performance involves measuring when foragers decide to move to a new "patch" with a higher yield. We assessed this using Anne Treisman's famous feature versus conjunction manipulation in an iPad foraging task. We measured how well patch leaving accorded with the predictions of the marginal value theorem that describes how foragers may optimize their foraging by leaving a patch once the average yield within a patch drops below the average yield in the whole environment. Human foraging in our paradigm deviated from the predictions of such optimal foraging conceptions, and our participants kept on foraging within the same patch for longer than expected. Patch leaving and intertarget times differed surprisingly little between feature and conjunction foraging, especially in light of the dramatic differences typically seen between performance on feature and conjunction visual search tasks. Other aspects of foraging performance (run number and switch costs) differed strongly between feature and conjunction foraging, however. We conclude that human foraging is probably influenced by too many factors to be captured with a relatively simple mathematical model.
\end{abstract}

Keywords Attention · Attention: Selective $\cdot$ Cognitive and attentional control

A common model of visual attention involves the well-known visual search task (Egeth et al., 1972; Neisser, 1963, 1964; Treisman et al., 1977). In the most common version of the task, observers search for a single predesignated target that is either present or absent among distractors (see Kristjánsson, 2015; Wolfe \& Horowitz, 2017, for reviews). This task has, for many years, been a source of important information about how we orient in the visual world and interact with it, and the task plays a major role in many prominent theoretical accounts of visual attention (Duncan \& Humphreys, 1989; Eckstein, 1998; Palmer, 1994; Verghese \& Nakayama, 1992; Wolfe, 1994), including Anne Treisman's well-known feature integration theory (Treisman

Árni Kristjánsson ak@hi.is

1 Faculty of Psychology, School of Health Sciences, University of Iceland, Reykjavík, Iceland

2 School of Psychology, National Research University Higher School of Economics, Moscow, Russian Federation
\& Gelade, 1980; Treisman, 1988; see review in Kristjánsson \& Egeth, 2019).

But despite their undeniable value, such single-target paradigms may not capture attentional function in richer environments that can involve many targets to look out for among various sources of distraction. Modelling such situations experimentally may arguably be more appropriate for understanding behavior in natural visual environments.

Recently, researchers of vision and visual attention have therefore increasingly used studies of visual foraging for many targets of various types among many distractor types, instead of single-target visual search to investigate human attentional orienting (Biggs \& Mitroff, 2015; Cain, Vul, Clark \& Mitroff, 2012; Hills, Kalff \& Wiener, 2013; Kristjánsson, Jóhannesson \& Thornton, 2014; Kristjánsson \& Kristjánsson, 2018; Wolfe, 2013; Zhang, Geng, Fougnie \& Wolfe, 2017; see Kristjánsson, Ólafsdóttir \& Kristjánsson, 2019 for review). The reasoning has been that foraging tasks with multiple targets of various types provide a more detailed picture of the function of visual attention. Many species highly related to humans perform such foraging tasks for large portions of their lives as they gather food, so it is not unreasonable to assume 
that such tasks have shaped our visual system through natural selection and that foraging can provide information about how humans orient in their visual environments. The literature on foraging has in fact developed within the literature on animal behavior and many important insights have been provided by these studies on animals (Dawkins, 1971; Dukas, 2002; Tinbergen, 1960; Bond, 2007; Langley et al., 1995).

We have recently developed a foraging task where observers can select multiple targets of two (or more) different types by tapping them with their fingers (Kristjánsson, Jóhannesson \& Thornton, 2014; Kristjánsson \& Kristjánsson, 2018). Once the targets have been tapped, they disappear, and the trial ends when all the targets have been tapped and are gone. In this task we have manipulated attentional load by using a classic feature versus conjunction manipulation, made famous in Anne Treisman's seminal studies (Treisman, Sykes \& Gelade, 1977; Treisman, 1977; Treisman \& Gelade, 1980). The typical visual search finding is that when a search target is separable from distractors on a single feature, processing of the search stimuli is faster, resulting in shorter reaction times than when they can only be distinguished from distractors by a conjunction of features (Treisman et al., 1977). The foraging task and the manipulation of features versus conjunctions has yielded many novel insights into the nature of visual orienting (e.g. Kristjánsson, Jóhannesson \& Thornton, 2014; Kristjánsson \& Kristjánsson, 2018; Kristjánsson, Thornton \& Kristjánsson, 2018; Kristjánsson, Thornton, Chetverikov \& Kristjánsson, in press).

In our studies observers have had to forage until all targets have been tapped and are gone. The foraging trial then ends, and observers proceed to the next trial. The paradigm where observers finish all targets on the screen and only then move to the next display yields many informative variables (see overview in Kristjánsson, Ólafsdóttir and Kristjánsson, 2019). Firstly, run number denotes how often participants switch between target types and has been found to depend on task demands (Dukas \& Ellner, 1993; Kamil \& Bond, 2006; Kristjánsson et al., 2014). Secondly, intertarget times can be measured, which involve the time that lasts between one tap on a target and the next tap on a target. The paradigm also allows the measurement of switch costs that denote the slowing of taps on targets that occurs when observers choose a different target type than they did on the last trial and end peaks in intertarget times that reflect the long time that observers typically take to locate the last target on the screen (the trial ends when all targets have been tapped).

\section{The concept of optimal foraging}

But an important variable that determines attentional orienting in the wild is that foraging animals may not be confined to a single source of food, but can move to a new food source when the availability within the current source becomes low.
An ecologically more valid paradigm may therefore involve allowing observers to move to another source of targets when targets are few or hard to find in the current environment, without having to finish all targets.

The procedure that we have used, where observers have essentially unlimited time to tap all the targets may also have some drawbacks from a theoretical perspective. Animal or human foragers probably do not typically stick around the same food source until all targets are finished. Consider berry picking. You may decide to start picking berries from a highyield berry bush, but as you pick, fewer and fewer berries are available in the bush and it takes time and effort to finish them all. So, to maximize your acquisition rate it may make sense to move to a berry bush that has more targets before you have finished collecting all in the current one, if it is not too far away or hard to get to (see Wolfe, 2013).

An influential account of foraging behavior is the so-called optimal foraging theory (Pyke, Pulliam \& Charnov, 1977; Hills, 2006; Stephens, Browne \& Ydenberg, 2007). Optimal foraging theory assumes that organisms should, through natural selection or within-lifetime learning, arrive at the best strategy to maximize their intake of food at the lowest energy expenditure. Optimal foraging theory has its roots in studies on how animals forage, or feed. From seminal work on how animals organize their foraging (e.g. Mook, Mook \& Heikens, 1960; Tinbergen, 1960) to theoretical accounts of optimal foraging (e.g. Charnov, 1976; Pyke, Pulliam \& Charnov, 1977) researchers have investigated and tried to determine which parameters and factors shape the foraging patterns of animals.

A key concept in this literature is the notion of the rate of intake of food or targets, or in other words the acquisition rate, typically involving how many targets are collected per second. At the heart of many theories of optimal foraging is the marginal value theorem (MVT, Charnov, 1976), which assumes that observers have implicit awareness of the average collection rate within the environment and they will continue to forage within a certain food source ("patch") until the acquisition rate (that depends on how many food items are available) drops below the average rate in the environment and another richer source is available. Foragers will try to maximize their energy intake, while minimizing their energy output, and do so using MVT (Charnov, 1976; Bartumeus \& Catalan, 2009). Note also that according to MVT, patch leaving should be influenced by the cost (in time or effort) of moving to another patch. Foragers should on average stay longer in a patch as travel time between food sources becomes longer or as the energy required for moving increases.

The application of these concepts allows the modelling of the optimal time spent in a patch depending on the number of targets available in the current versus an alternative patch and the cost of switching between patches. Earlier data seemed to support optimal foraging theory and human and animal foraging behavior seemed to roughly fit its predictions (Cain et al., 
2011; Hutchison, Wilke \& Todd, 2009; Pyke, 1978a; Kamil, Yoerg \& Clements, 1988). One example is how Pyke (1978b) found that the decisions of hummingbirds on when to leave a food source was determined by the number of flowers visited, the number of flowers available, and how much food was available at the last food source they visited. Partial support for optimal foraging theory in human foraging has also been found (e.g. Cain et al., 2011), even though with increased setsize (Cain, Boettcher \& Wolfe, 2014) and with differing value and target proportion, results deviated from the predictions of optimal foraging theory (Soce, Cain, \& Wolfe, 2016).

It is important to note, however, that if models that incorporate the marginal value theorem are supposed to apply to how humans or animals forage in the environment, this entails the assumption that their decision making is rational and calculated. Some have argued that animals and humans are actually not optimal foragers and that their behavior is far more random than such theoretical approaches imply (Pierce \& Ollason, 1987). And discrepancies from the predictions of MVT have indeed emerged. Currently most researchers accept that humans (and other animals) are not strictly optimal foragers and several different factors determine foraging behavior (Wolfe, 2013) such as attentional load and difficulty (Dukas \& Kamil, 2001; Bond, 1982; Staddon \& Gendron, 1983). Wolfe (2013) used a patch-leaving design, involving mouse clicks on a computer screen, with manipulations of patch quality, such as the number of berries, or how valuable the berries were, involving distinguishing "good" berries from "bad" ones, finding deviations from MVT predictions and Wolfe concluded that many different rules may be applied that are determined by the specific conditions of each task (see general discussion).

Overall, foraging tasks have yielded a picture of attentional orienting that is possibly more dynamic and arguably more interesting than single-target search tasks. In fact, a recent provocative interpretation is that single-target searches can simply be described as the last target selection in an exhaustive foraging task. These last selections yield, in essence, the same RT patterns as single target feature and conjunction searches (Kristjánsson, Thornton, Chetverikov \& Kristjánsson, 2020).

\section{Current aims}

Using our previously developed foraging paradigm on iPad touch screens, we investigated when observers choose to leave a 'patch' and move onto the next one, and how this interacts with task difficulty, as reflected in Anne Treisman's classic feature vs. conjunction attentional load manipulation (targets defined by a feature difference versus targets defined by a conjunction of features). Our paradigm therefore differed from our previous designs in that a button was present on the screen that allowed observers to switch to a new patch where the percentage of targets was $50 \%$, when they chose, instead of needing to finish all targets before moving to the new patch. The predictions of conceptions such as the marginal value theorem (that is at the heart of optimal foraging theory), dictate that observers will choose to move to the next patch when the average acquisition rate within each patch drops below the average acquisition rate in the environment (see Figure 1). In other words, if the average acquisition rate in the task is 1 target per second, observers should move on to the next patch once their acquisition rate drops below 1 target per second. But for the orienting of attention we may ask whether we usually are in situations that involve straightforward applications of optimal foraging theory or the marginal value theorem?

In the current task, the next patch is a single tap away. Given the short "travel time" between patches there is little cost to moving to a new one. OFT therefore predicts that foragers should leave the patch at the first hint of a drop in collection rate (see Figure 1). Note that the costs of moving to a new patch are not manipulated in this study. Another important feature of the task in experiment 1 is that the targets on the screen did not disappear once they were tapped but stayed on the screen. We did this to increase the difficulty of the task in an effort to exaggerate differences between the paradigms, but also to investigate the potential effect of memory for tapped locations, since observers should avoid re-tapping already tapped targets which would be a wasted opportunity for point collection (see methods). If the items stay on the screen and do not disappear when tapped, observers must memorize the tapped targets to maximize their yield (see Thornton \& Horowitz, 2004 and Cain \& Mitroff, 2013 for discussion of this issue). One question that we wish to address here is whether the attentional load of the task will affect memory for tapped targets as revealed by re-taps on already tapped ones. Additionally, we wondered how run behavior would be affected by the patch leaving manipulation and whether there would be an interaction with the attentional feature versus conjunction manipulation. We also measured intertarget times throughout foraging trials and switch costs that occur when observers select a different target type from their last selection. In experiment 2 we then test a paradigm where the targets disappear once tapped for comparison, and in experiment 3 we test performance with less severe penalties for erroneous taps on distractors than in experiments 1 and 2.

\section{Experiment 1}

\section{Methods}

Participants Eighteen volunteers, from the University of Iceland and the Icelandic Center for Treatment of Anxiety 


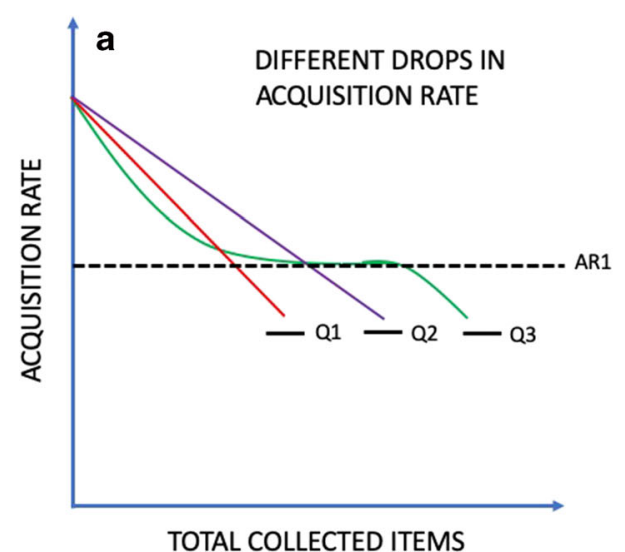

Fig. 1 Predictions for when observers stop foraging within a given patch (or food source) as a function of acquisition rate and total collected items according to the marginal value theorem. A) Different drops in acquisition rate predict different quitting points (Q1, Q2 and Q3), that all involve when the rate drops below the average rate in the environment.

Disorders ( 7 female), 18-61 years old $(M=32.95)$ participated, they received gift cards from a local shopping center for participation. All had normal or corrected-to-normal vision. Prior to data collection, all participants gave written informed consent and all aspects of the study were in accordance with the requirements of the local ethics committee.

Equipment The stimuli were displayed on an iPad 2 with screen dimensions of 20 by $15 \mathrm{~cm}$ and an effective resolution of 1024 by 768 pixels. The iPad was placed on a table in front of participants in landscape mode, so that viewing distance was approximately $60 \mathrm{~cm}$. Stimulus presentation and response collection were carried out with a custom iPad application written in Swift using Xcode.

Stimuli During conjunction foraging, the targets were red squares and green disks and the distractors were green squares and red disks for half the participants. For the other half, this was reversed (see Figure 2). During feature-based foraging, the targets were red and green disks while the distractors were yellow and blue disks for half the participants but reversed for the other half of the observers. There were 80 stimuli on the screen at the start of each trial, 20 stimuli of each type, 40 targets and 40 distractors. Their diameter was 20 pixels (approximately $0.37^{\circ}$ ). The items were randomly distributed across a nonvisible 10 by 8 grid that was offset from the screen edge by 150 by 100 pixels. The whole viewing area therefore occupied 15 x $12 \mathrm{~cm}$ (approximately $14.3 \times 11.4^{\circ}$ ). Gaps between rows and columns ensured that items never approached or occluded one another. The overall spatial layout and location of targets and distractors was generated independently on every trial.

Procedure The experiment was run in a small quiet room with mild overhead lighting but no reflectance from the iPad

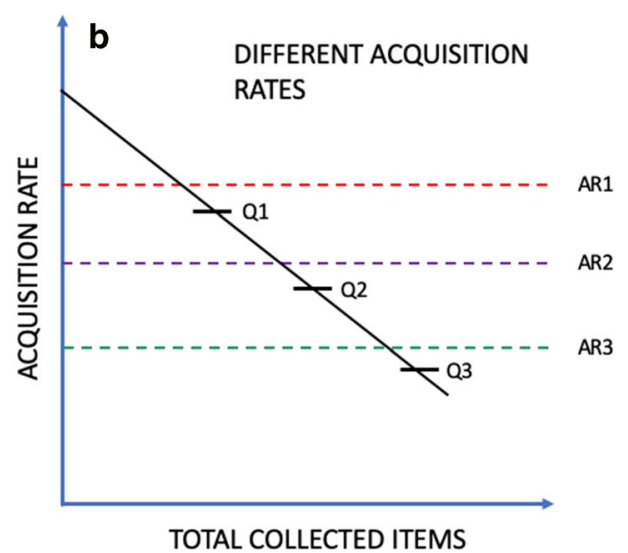

B) If the average acquisition rates (AR1, AR2 and A3) differ between environments the quitting points should differ accordingly for the same drop in acquisition rate. For simplicity, this figure does not take the cost of moving between patches (in time or effort) into account.

screen. On each trial, participants were instructed to tap targets as quickly as possible using the index finger of their dominant hand. A counter at the bottom of the screen indicated the number of completed trials. Importantly, participants had the choice of pressing a button any time they wanted to get a new full display (i.e. patch leaving). Once the items had been tapped they remained on the screen and observers were encouraged to try to remember which parts of the display they had already tapped. The participants had 60 seconds to tap as many targets as possible.

Participants received a point for each tapped target. They did not, however, receive any points if they re-tapped targets. Participants were told to collect as many points as possible within the 60 second trial time. After each trial, the total score for the trial was displayed along with the highest score for each participant.

Participants started each experimental block by pressing a "play" button on the screen when ready and the stimuli then appeared. They were instructed to finish 16 trials and let the experimenter know when they had finished. Participants started with 2 practice trials, to familiarize themselves with the iPad and the stimuli and to get a feel for how sensitive the touch screen was to the tapping. They were told that they would complete 2 blocks of 16 trials, 32 trials in total, and that they could take a break between the blocks. If participants tapped a distractor, the trial ended, and they received an error message. They could start a new trial by pressing the play button. The order of the conditions was counterbalanced across participants so that half of the participants started with feature foraging and half with conjunction foraging.

Participants were told which two target categories they were to select. The scoring was explained (1 point for each target, 0 points for tapping a previously tapped target), and that they could get a new display with 80 new stimuli (50\% of which, would be targets) by pressing the arrow to the right of 

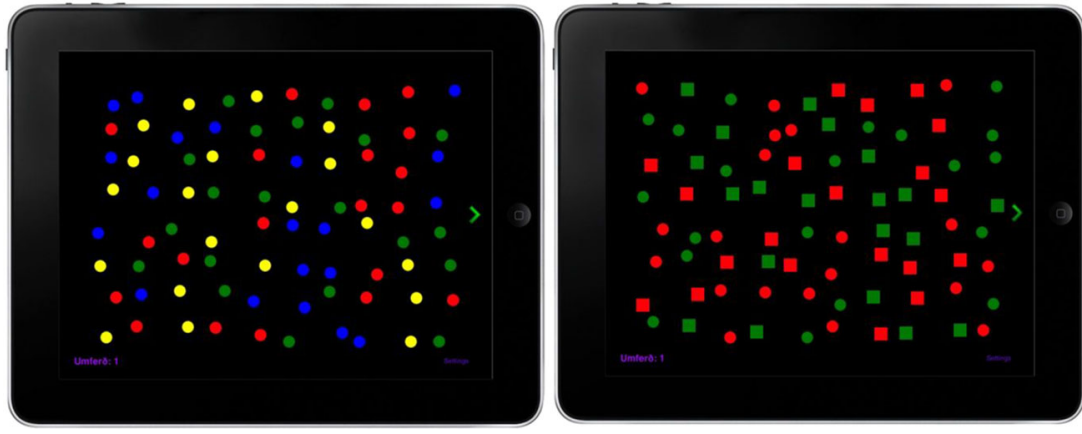

Fig. 2 Examples of foraging trials. Feature foraging on the left and conjunction foraging on the right. By clicking on the arrow at the right of the search displays, observers could move to a new patch.

the display at any time and they could do this as often as they wanted during the 60 seconds. They were told that they should collect as many points as possible and try to beat their highest score (which was displayed below the current trial score between trials). They were then told that tapping a distractor would result in the termination of the trial and that they would have to redo the whole trial. The experimenter remained in the room during practice trials and the participants could ask questions, if participants did not press the patch leaving button during the whole trial in either of the practice trials, they were reminded that they could press the button at any time to get a fresh display of stimuli. This only happened once during the running of the experiment.

Data Analysis The data were cleaned by erasing taps that were not on any stimuli (10,456 taps, $10 \%$ of total taps). Then any trial that ended with an error was deleted. This left 576 trials in the final dataset. The independent variables in the analyses were condition (feature vs. conjunction foraging), $\mathrm{N}$ from termination (the number of taps before participants moved to a new patch) and type of tap (repeated selection from the same target category or a switch to the other target category). The dependent variables were the length of a run, intertarget times (ITTs) and the collection rate (the number of targets collected per second). A run is defined as a sequence of repeated selections from the same target group, and directly reflects the number of switches between target categories. One switch translates to two runs, 10 switches mean 11 runs, and so on.

The ITT's measure the duration between taps on two successive targets. One potential reason for the large differences in run behavior during foraging in previous studies is that switching between target types entails a cost, and that this cost is higher during difficult foraging tasks. Participants may therefore rarely switch during conjunction foraging to maximize speed (see Jóhannesson et al., 2016 and Ólafsdóttir et al., 2016). Switch costs can be assessed by measuring how ITTs differ by whether observers tap the same targets as they last did, or switch to the other target type. Examining the pattern of ITTs, particularly with respect to switch costs, can therefore shed light on foraging strategies. For the repeated-measures analyses of variance (ANOVAs), Greenhouse-Geisser corrections to the degrees of freedom were used to correct for any violations of sphericity.

\section{Results and discussion}

\section{Mean collection rate}

Figure 3 shows how the mean collection rate per second changes as a function of the temporal position of each selection within trials, or in other words how close a given trial is to the moment when the observers decide to leave the patch and move to the next one, presented separately for feature and conjunction foraging. The first thing to note is that the two types of foraging

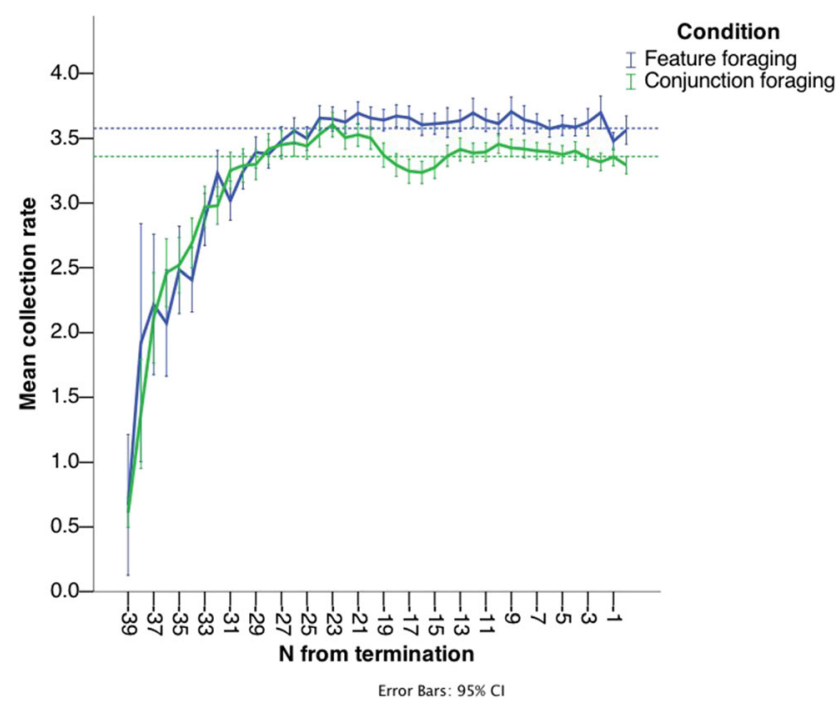

Fig. 3 Mean collection rate per second (on the ordinate) as a function of the number of taps from termination of the trial (or when observers "left a patch") on the abscissa. The rightmost data point denotes the last target before observers moved to the next patch. Note that for the highest $\mathrm{N}$ from termination of the foraging trial, there are very few target selections behind each average acquisition rate as these would be from trials where observers finished all or most of the targets on the screen which did not happen often (see Table 1). The dotted lines represent the average collection rate in each condition. The error bars denote $95 \%$ confidence intervals 
task converge on different mean collection rates for when observers choose to leave the patch. This rate is higher for feature foraging (3.58 targets) than for conjunction foraging (3.36 targets). This means that observers tend to be willing to accept a slightly lower acquisition rate during conjunction than feature foraging. Secondly, for conjunction foraging, there is a notable dip in the collection rate for targets ca. 12 to 21 from termination that notably only occurs during conjunction foraging. The most likely reason for this is that this reflects costs of switching between categories, that slow down the collection rate. This switch during conjunction foraging can be seen in the midpeaks in Figure 4 that shows the switch rates as a function of the position within a selection sequence.

Figure 4 shows the proportion of switches as a function of condition and position within a trial. These results can partly explain the patterns seen in Figure 3. There are far more switches for the positions within trials that correspond to the dip in Figure 3 for conjunction foraging than for other positions within the trial (except for the first 3-4 trials). The figure also shows that the proportion of switches is rather constant throughout trials for feature foraging. When we analyzed the collection rates to confirm that collection rates are different based on $\mathrm{N}$ from termination, only the last 20 targets in each patch were analysed. This was done since very few trials were behind the most extreme $\mathrm{N}$ from termination numbers (towards the left in figure 3). A 2 (condition) X 20 (N from termination) repeated measure ANOVA shows that the difference in collection rates between conditions is significant $\left(\mathrm{F}(1,17)=15.53, p<.001 \eta_{p}^{2}=.477\right)$, the $\mathrm{N}$ from termination $\left(\mathrm{F}(6.39,108.60)=2.72, p=.015 \eta_{p}^{2}=.138\right)$ and a significant interaction between condition and $\mathrm{N}$ from termination $\left(\mathrm{F}(6.21,105.62)=2.69, p=.017 \eta_{p}^{2}=.137\right)$. This interaction reflects the dip in collection rates seen for conjunction

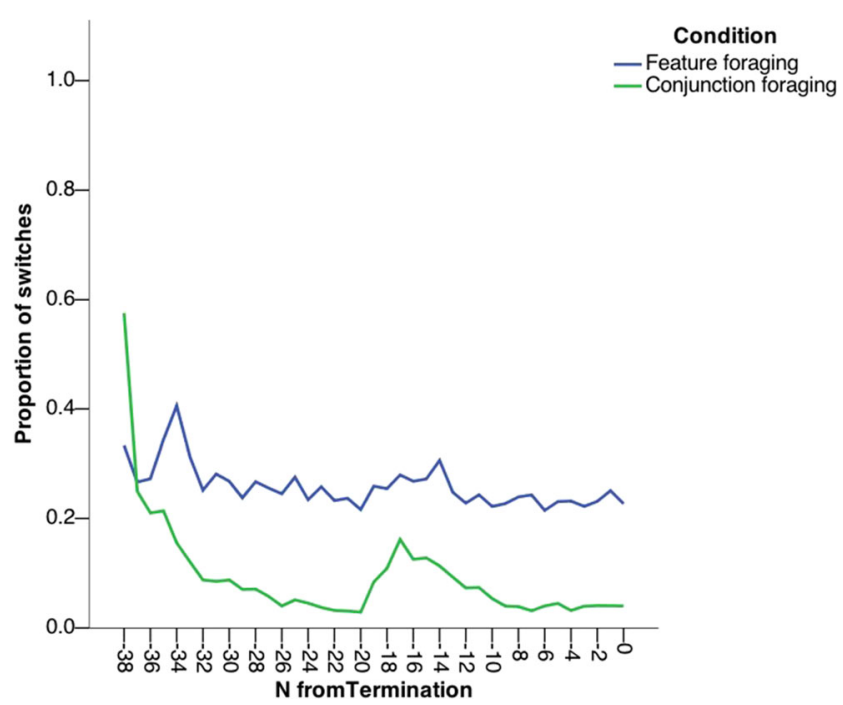

Fig. 4 The proportion of switches as a function of trials from when observers left the patch (on the abscissa), shown for feature (blue) and conjunction (green) foraging foraging that occurs as observers tend to switch between target types (Figure 3).

With regard to conceptions of visual foraging, one highly interesting aspect of the results is that the average acquisition rate does not decrease during the trial and stays quite constant. This does, on the surface, not fit well with predictions from optimal foraging theories that incorporate the marginal value theorem, since according to the MVT, observers should tend to stop foraging when the acquisition rate falls below the average rate. But note also that there is no decrease in acquisition rate throughout the foraging trials except for the small dip when observers switch from one target category to the other during conjunction foraging. This could be thought to reflect the characteristics of this particular foraging task since it may be too easy to reveal decreases in collection rate. But this explanation does not seem to be quite satisfactory. If difficulty plays a role, it is surprising how similar the pattern is for feature and conjunction foraging, given the difference in difficulty between those two conditions. This pattern may also reflect that in our design the items did not disappear, a point that we address in experiment 2. But we should also note that the low acquisition rates at the beginning of trials presumably reflect that observers are slow in initiating the foraging, until they enter what we have termed the "cruise-phase" (Kristjánsson et al., 2020). Interestingly, this is similar for feature and conjunction foraging.

\section{Run lengths}

Figure 5 shows the average run lengths for feature and conjunction foraging, confirming that observers tend to switch

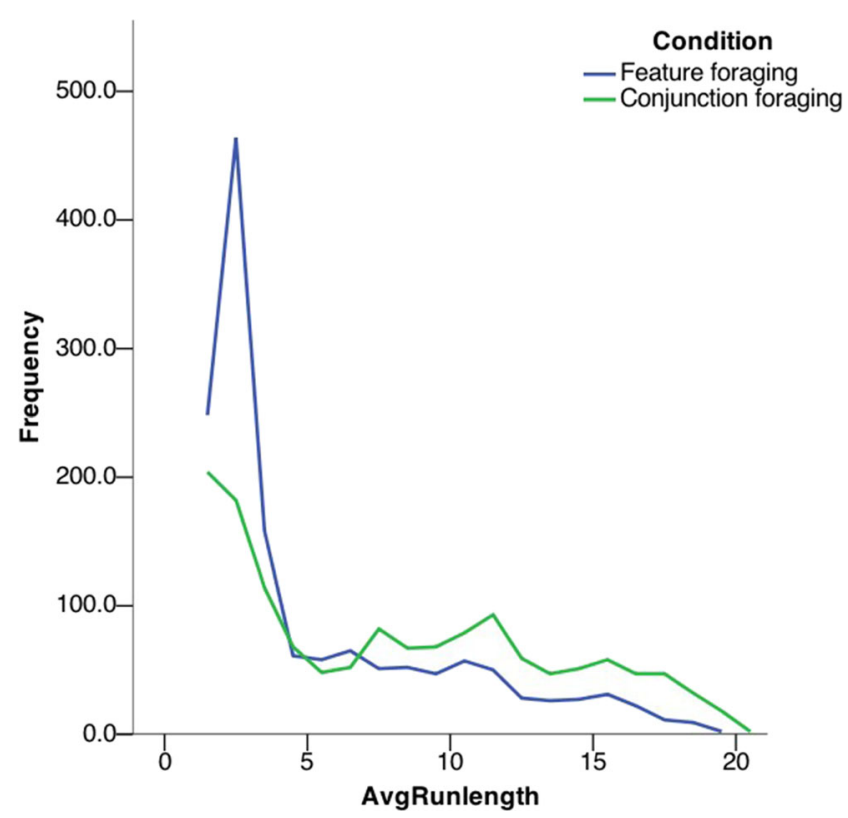

Fig. 5 The average run lengths per trial for the feature and conjunction foraging conditions 
more often between target types during feature than conjunction foraging. The figure shows that there are far more short runs for feature than conjunction foraging. This is consistent with what we have found in previous studies (Kristjánsson et al., 2014; Jóhannesson et al., 2017): Average run number is low for feature foraging while observers switch much less during conjunction foraging, indicating that keeping two conjunction templates in working memory is much harder than two feature-based templates (see Kristjánsson \& Kristjánsson, 2018). A paired-samples t-test between the average run length for each participant between feature and conjunction foraging confirmed a significant difference $(t(17)=-4.23, p<.001)$. Notably this also means that the two manipulations that differ in this study from previous studies (patch leaving and that targets remain on the screen once they have been tapped) did not affect the basic patterns for run lengths seen in our previous studies.

\section{Switch costs}

Interestingly, as Figure 6 shows, if the same target is selected as on the previous trial, intertarget times are almost identical for feature and conjunction foraging, while switching between different target types takes much longer during conjunction foraging (shown by the large switch costs). A 2(condition) X 2 (switch/repeat) repeated measures ANOVA confirmed a significant effect of condition $\left(\mathrm{F}(1,17)=54.04, p<.001 \eta_{p}^{2}=\right.$ $.761)$, switch $\left(\mathrm{F}(1,17)=68.44, p<.001 \eta_{p}^{2}=.801\right)$ and a significant interaction between condition and whether the tap was a repeat or switch $\left(\mathrm{F}(1,17)=42.51, p<.001 \eta_{p}^{2}=.714\right)$. The fact that selection times are similar between the 2

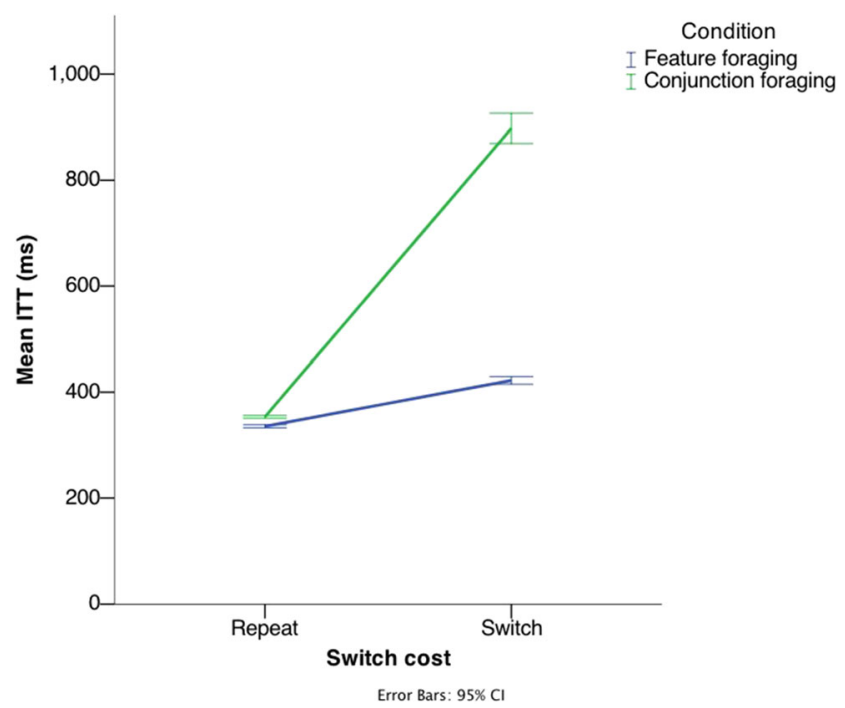

Fig. 6 Intertarget times for feature and conjunction foraging as a function of whether observers select the same target type as on the last trial (repeat) or select the other target type (switch). The differences between ITT's for repeats versus switches denote the switch costs. Error bars denote $95 \%$ confidence intervals conditions, when no switches occur, may appear surprising in light of well-known findings on feature and conjunction search. We speculate however that priming effects are likely to play a role during conjunction foraging as observers repeatedly select the same target type (see e.g. discussion in Kristjánsson et al., 2014; Wolfe et al., 2018) but further experimentation is needed for firm conclusions on this point.

\section{Patch leaving and revisits of previously tapped locations}

Our current design contained two changes from our previous studies with this iPad foraging task. Firstly, observers had the opportunity to leave the foraging display (or 'patch') that they were currently in and immediately get a new display where the availability of targets was 50\%. A second change was that the items did not disappear once they had been tapped. This presumably entails demands upon memory for already checked locations since under the point collection scheme observers would waste their time by re-tapping already tapped targets.

Table 1 shows the average number of patches that observers went through in the 60 seconds that they had for each trial. Observers seemed to get through a slightly higher number of patches during feature foraging, perhaps not surprisingly, since the intertarget times are overall lower for feature foraging (see Fig 5). Their average total collection rates are 132.8 targets during feature foraging and 124. 9 targets for conjunction foraging and this difference is surprisingly small. Within each patch, observers tap just under 27 targets per patch on average (leaving 13 targets), and the difference between feature and conjunction foraging is only 0.31 targets per patch, showing little or no difference in how exhaustively observers forage between conditions. What this essentially means is that observers can rather effectively compensate for the larger difficulty for conjunction foraging by repeatedly selecting the same target type, and as mentioned before, potentially utilize priming effects. Three paired-sample t-tests were conducted to see whether the number of patches, targets per patch or the proportion of revisits differed between conditions. There was no significant difference between the number of patches or targets per patch between the two foraging conditions ( $p=.419$ and $p=.740$ respectively). The difference between the proportion of revisits, despite being almost three times higher for feature foraging was not significant, $t(17)=$ $1.66 p=.116$, which means that in light of the variability in

Table 1 Summary of the number of patches visited, targets collected and re-taps on already selected targets

\begin{tabular}{|c|c|c|c|}
\hline \multicolumn{2}{|l|}{ Avg. Patches } & \multirow{2}{*}{$\begin{array}{l}\text { Avg. Targets per patch } \\
26.93\end{array}$} & \multirow{2}{*}{$\frac{\text { Avg. percentage of revisits }}{3.35}$} \\
\hline Feature & 4.93 & & \\
\hline Conjunction & 4.69 & 26.62 & 1.25 \\
\hline
\end{tabular}


these numbers the actual differences were small. Overall, revisits were surprisingly few, which suggests that observers' memory for visited items was rather good. This can partly be explained by the way participants forage, which is not random, but rather they tend to forage rather systematically through the display. A bivariate correlation was calculated for each trial in each condition for each participant, where the correlation between target number a position on $\mathrm{x}$ and $\mathrm{y}$ coordinates on the screens were calculated, and the higher correlation was selected as best-R to indicate systematicity of the foraging path (see Woods et al., 2013). The foraging path was more systematic for feature foraging with the average best- $\mathrm{R}=$ .535 with $81 \%$ of trials having a higher correlation with the $\mathrm{x}$ axis target positions, meaning participants search up and down repeatedly (resulting in lower correlation with y-axis values) but in general, in a more systematic sweep from left to right or right to left. For conjunction foraging the best-R was $=.286$ with $65 \%$ of trials having a higher correlation with $\mathrm{x}$-axis values. In both conditions, these correlations are significant, indicating systematic foraging. This is not surprising, as both animals and humans have been shown to forage systematically (Baum, 1987; Woods et al., 2013). Some researchers have, however, shown that reading-pattern like foraging behavior decreases when stimuli are moving (Cain et al., 2014).

\section{Experiment 2}

One possible explanation for the deviations from MVT in Experiment 1 is that, unlike most previous studies on human foraging, the targets in Experiment 1 did not disappear once they were selected. Our reason for using this manipulation was that it allowed us to study whether observers revisit previously tapped locations. This change in task, might however affect performance and be the reason for any deviation from the predictions of MVT. Note however that run numbers and intertarget times did not deviate much from previous results. But because of this we repeated Experiment 1, except that targets disappeared when tapped, in line with many previous human foraging tasks. It is possible that if the number of visible targets is constant even though observers tap targets that this affects their foraging strategies. The main goal in experiment 2 was therefore to measure whether patch leaving behavior would change from Experiment 1.

\section{Method}

The methods for Experiment 2 were identical to Experiment 1 with the exception of the changes listed below.

Participants Eight participants ( 4 female), between the ages of 23-34 ( $\mathrm{M}=28.4)$ participated in the experiment. They received no payment for participation. In light of the stability of the results of the previous study we reasoned that this lower number of participants would suffice for this follow-up experiment.

Procedure The only change from Experiment 1 involved what happened when a target was tapped. When a target was tapped, instead of remaining on the screen, it disappeared, reducing the total number of stimuli on the screen by one. Otherwise, the procedure was the same as in Experiment 1.

\section{Results}

Figure 7 shows clearly that observers forage similarly to Experiment 1 even though the targets disappeared once they had been tapped. The average number of targets tapped per patch was 26.4 targets during feature foraging and 29.6 targets during conjunction foraging. A 2 (condition) X 20 (N from termination) repeated measure ANOVA revealed no significant difference in collection rates between conditions $(\mathrm{F}(1,7)=1.19$, $\left.p=.311 \eta_{p}^{2}=.145\right)$, but the difference between the $\mathrm{N}$ from termination was significant $\left(\mathrm{F}(2.40,16.81)=3.74, p=.039 \eta_{p}^{2}\right.$ $=.348)$ and there was a significant interaction between condition and $\mathrm{N}$ from termination $\left(\mathrm{F}(2.73,19.12)=6.01, p=.006 \eta_{p}^{2}\right.$ $=.462$ ). This interaction presumably reflects the dip in collection rates seen for conjunction foraging that occurs as observers switch between target types (Figure 7). The marginal value theorem predicts that observers should leave the patch once the acquisition rate falls below the average in the display. But in our results the acquisition rate stays roughly constant for a long time. Interestingly, the difference between feature and conjunction foraging was not significant in Experiment 2. But most

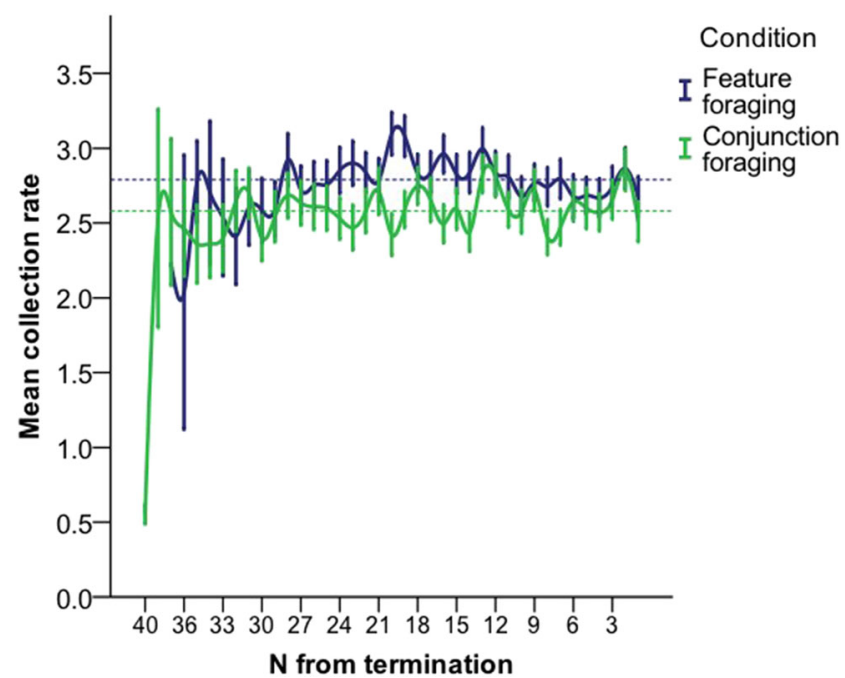

Fig. 7 Mean collection rate per second (on the ordinate) as a function of the time from termination of the trial (or when observers "left a patch") on the abscissa. The dotted lines represent the average collection rate in each condition 
importantly, the fact that the participants collection rates often drop below the average collection rate in conjunction foraging, before they leave the patch makes clear that the reason for the performance patterns in Experiment 1 was not simply that the targets did not disappear once tapped.

Figure 8 shows the average run lengths for feature and conjunction foraging, showing that as in Experiment 1, observers tend to switch more between target types during feature than conjunction foraging and there are thus far more short runs for feature foraging than conjunction foraging, again consistent with previous findings (Kristjánsson et al., 2014; Jóhannesson et al., 2017). A paired-samples t-test between the average run length for each participant between feature and conjunction foraging confirmed a significant difference $(t(7)=-5.33, p<.001)$.

\section{Switch costs}

As in Experiment 1, if the same target is selected as on the previous trial, intertarget times were almost identical for feature and conjunction foraging, while switching between different target types took much longer during conjunction foraging, shown by the large switch costs for conjunction foraging (see Figure 9). A 2 (condition) X 2(switch/repeat) repeated measures ANOVA confirmed a significant effect of condition $\left(\mathrm{F}(1,7)=112.09, p<.001 \eta_{p}^{2}=.941\right)$, switch $(\mathrm{F}(1,7)=$ $\left.809.11, p<.001 \eta_{p}^{2}=.991\right)$ and a significant interaction between condition and whether the tap was a repeat or switch $\left(\mathrm{F}(1,7)=268.45, p<.001 \eta_{p}^{2}=.975\right)$.

\section{Discussion - experiment 2}

Making the targets disappear once they had been tapped, did not change the results from Experiment 1. The collection rates remained very similar between feature and conjunction foraging and as in experiment 1, participants did not leave the patches despite dips in acquisition rates. Run length and switch costs remained comparable with Experiment 1 (confirmed by statistical tests). What this seems to show, is that the fact that the targets did not disappear, cannot explain the results from Experiment 1.

\section{Experiment 3}

Another possible explanation for our results in experiment 1 and 2 and how they seemingly deviate from the predictions of optimal foraging accounts, involves the large consequences of making an error. In experiments 1 and 2 , if the observers tapped a distractor, they had to start the trial over if they made an error. This might push participants towards a 'play-it-safe' strategy,

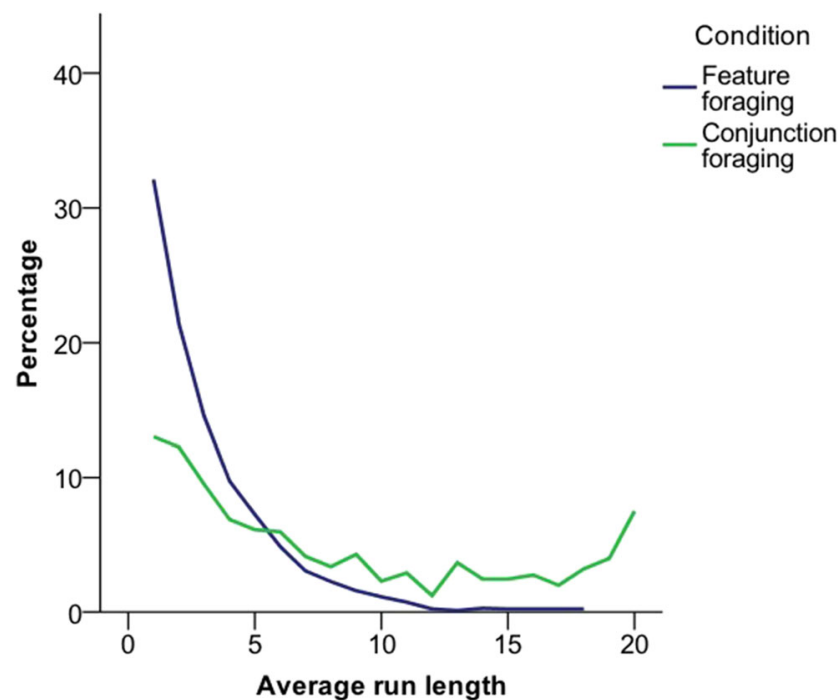

Fig. 8 The average run lengths per trial for the feature and conjunction foraging conditions in experiment 2

laying greater emphasis on avoiding mistakes than speed and foraging efficiency. This might explain the low number of runs during conjunction foraging and might contribute to the patch leaving pattern. To study this possibility, Experiment 1 was repeated, but now, errors did not result in the termination of the trial and observers having to redo it, but rather a point was subtracted for each error from the total score the participants received on the feedback screen after each trial.

\section{Method}

The methods for Experiment 3 were identical to Experiment 1 except for the changes listed below.

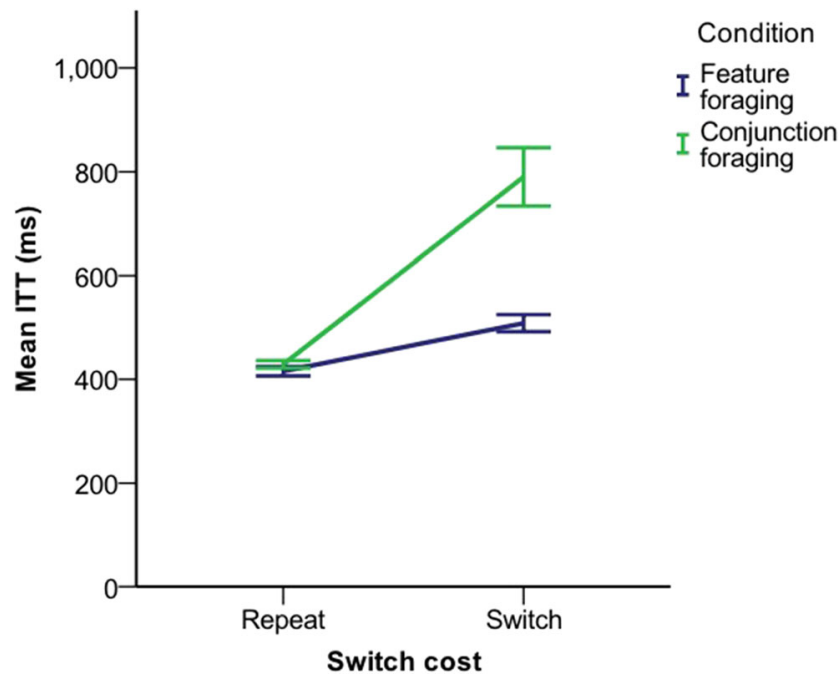

Fig. 9 Intertarget times in experiment 2 for feature and conjunction foraging as a function of whether observers select the same target type as on the last trial (repeat) or select the other target type (switch). The differences between ITT's for repeats versus switches denote the switch costs. Error bars denote 95\% confidence intervals 
Participants The same eight participants that participated in Experiment 2, participated in Experiment 3. Note that the order of experiments $2 \& 3$ was counterbalanced across participants. Again we note that the large power of experiment 1 prompted us to reason that fewer participants were needed for experiments 2 and 3 .

Procedure The procedure changed from Experiment 1, in the consequences of tapping a distractor. When a distractor was tapped, the trial score decreased by one but the trial continued as normal. Otherwise, all the procedures remained identical to Experiment 2, i.e. the distractors disappeared once tapped.

\section{Results}

Figure 10 clearly shows that observers foraging patterns in experiment 3 are similar to those in experiment 1 even though the penalty for tapping a distractor was reduced from trial termination and redoing the whole trial, to the less severe point reduction. This less severe penalty for errors did not affect the how exhaustively observers foraged. The average number of targets tapped per patch was 25.7 targets in feature foraging and 29.2 targets in conjunction foraging. As mentioned before, optimal foraging theories that include the marginal value theorem would predict that observers should move to the next patch once the collection rate drops below the average collection rate, but, as shown in Figure 10, this happens repeatedly and yet they keep on foraging within the same patch. This makes clear that the reason for the performance patterns in Experiment 1, where performance deviated from the predictions of the marginal value theorem was not that the penalty for errors was too high. As in Experiment 2, a 2 (condition) X 20 (N from termination)

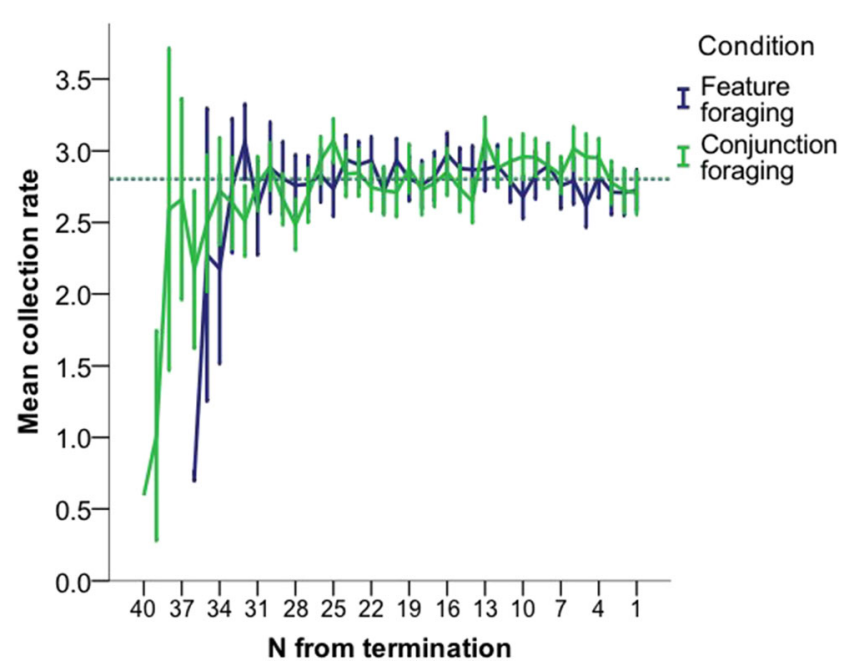

Fig. 10 Mean collection rate per second (on the ordinate) as a function of the time from termination of the trial (or when observers "left a patch") on the abscissa, in Experiment 3. Error bars denote 95\% confidence intervals repeated measure ANOVAs revealed no significant difference in collection rates between conditions $\left(\mathrm{F}(1,7)=.43, p=.535 \eta_{p}^{2}\right.$ $=.057)$, but the difference between the $\mathrm{N}$ from termination was significant $\left(\mathrm{F}(1.89,13.25)=6.39, p=.012 \eta_{p}^{2}=.477\right)$ and there was an interaction between condition and $\mathrm{N}$ from termination $\left(\mathrm{F}(2.01,14.08)=9.88, p=.002 \eta_{p}^{2}=.585\right)$.

Figure 11 shows the average run lengths for feature and conjunction foraging, revealing that as in Experiment 1, observers tend to switch more between target types during feature foraging than conjunction foraging and there are therefore far more short runs for feature foraging than conjunction foraging again consistent with previous findings (Kristjánsson et al., 2014; Jóhannesson et al., 2017). A paired-samples t-test comparing the average run length for each participant between feature and conjunction foraging confirmed a significant difference $(t(7)=-25.30, p<.001)$. Interestingly, the differences between the two foraging types are, if anything, stronger in Experiment 3 than in the previous two experiments.

\section{Switch costs}

As in experiments 1 and 2, if the same target was selected as on the previous trial, intertarget times were almost identical for feature and conjunction foraging, while switching between different target types took much longer during conjunction foraging (switch costs shown in Figure 12). A 2 (condition) X 2 (switch/repeat) repeated measures ANOVA revealed a significant effect of condition $(\mathrm{F}(1,7)$ $\left.=627.82, p<.001 \eta_{p}^{2}=.989\right)$, switch $(\mathrm{F}(1,7)=1087.08, p$ $\left.<.001 \eta_{p}^{2}=.994\right)$ and a significant interaction between condition and whether the tap was a repeat or switch $\left(\mathrm{F}(1,7)=616.76, p<.001 \eta_{p}^{2}=.989\right)$.

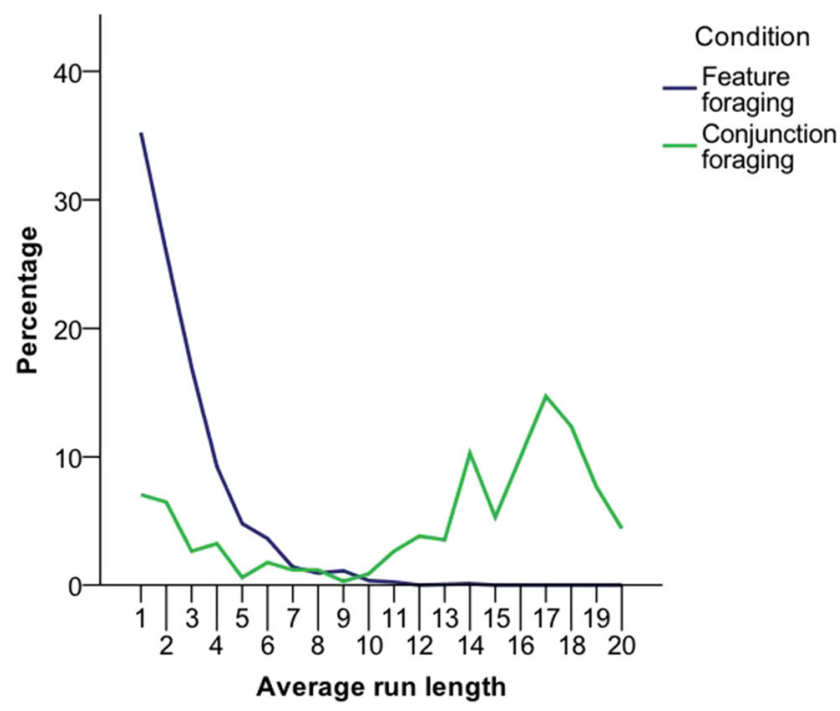

Fig. 11 The average run lengths per trial for the feature and conjunction foraging conditions in Experiment 3 


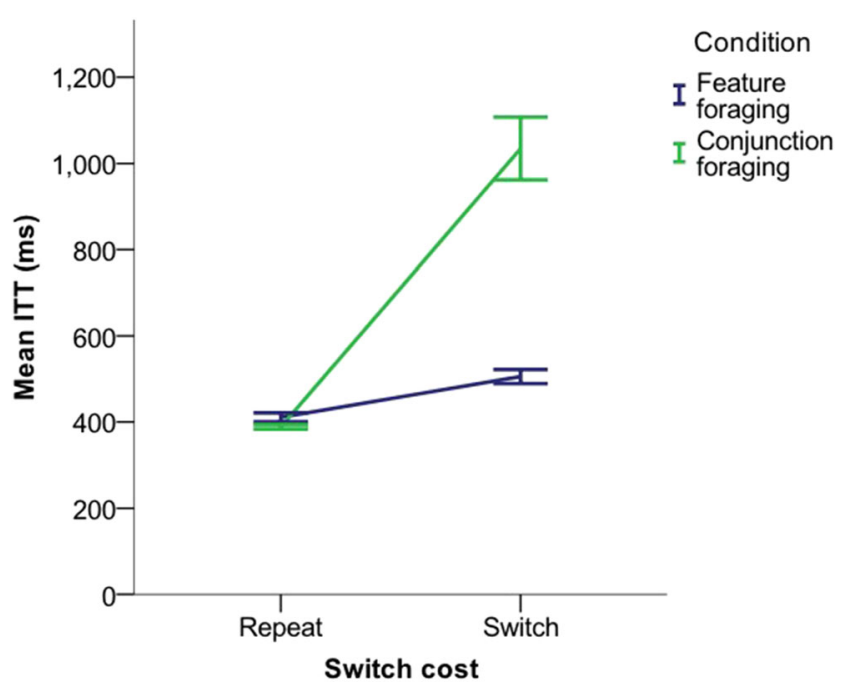

Fig. 12 Intertarget times in Experiment 3 for feature and conjunction foraging as a function of whether observers select the same target type as on the last trial (repeat) or select the other target type (switch). The differences between ITT's for repeats versus switches denote the switch costs. Error bars denote $95 \%$ confidence intervals

\section{Discussion - Experiment 3}

The results of Experiment 3 show that the results from Experiment 1 cannot be explained away as being the result of severe consequences of making an error (that the trial ends if a distractor is tapped). Even when the only penalty of making an error was point deduction the pattern was similar to that in experiment 1 . Neither can the results from experiment 1 be traced to the targets not disappearing after selection since in experiments 2 and 3 the targets disappeared, yet performance patterns were similar to what they were in Experiment 1. The difference in task conditions may make a difference, as can be seen in Experiment 3, where acquisition rates were similar between feature and conjunction foraging, but the difference between acquisition rates was, in any case, small in both experiments 1 and 2, and the main result patterns hold true throughout all three experiments presented here.

\section{General Discussion}

In this project we asked 4 main questions that we address in what follows:

Do observers organize their performance in accordance with the predictions of optimal foraging theory theories that include the marginal value theorem? The answer is clearly no. According to these conceptions observers should leave a patch once their acquisition rate drops below the average one. Instead, observers stayed within the same patch far longer than would be expected under MVT and kept on foraging for a long time while the acquisition rate stayed constant. And during conjunction foraging, as acquisition rates fell when observers switched between target categories, they tended not to move to a new patch. In fact, the total collection rates of targets were surprisingly similar for feature and conjunction foraging (see table 1).

Does allowing observers to move to a new patch when they want to ('patch leaving') affect foraging strategies? Overall, foraging strategies were similar to what we have seen with this iPad foraging paradigm where observers had to finish all targets in each foraging display, showing that allowing observers to switch to a new "patch" and keeping targets visible after they are tapped did not affect the strategies much. Their foraging still involved many switches between target types during feature foraging but long runs of selecting the same target type during conjunction foraging which means that run behavior was similar to what we have seen in previous studies.

Does foraging type affect patch leaving? Firstly, the average acquisition rate peaked at a higher rate for feature than conjunction foraging in experiments 1 and 2 but was similar in experiment 3 . The difference in experiments 1 and 2 seems to indicate that observers are willing to keep on foraging for a slightly lower yield during conjunction foraging than feature foraging (although this difference was not very large). Optimal foraging theories that involve the MVT cannot, at face value, account for this result, since patch leaving is not solely determined by the yield, nor the acquisition rate. Clearly the nature of the task in each case has a large effect upon how observers organize their foraging and what patch leaving strategies they use.

How does keeping targets on the screen after they have been tapped (presumably placing demands on working memory) affect foraging? Firstly, revisits to previously selected locations were quite few $(1-3 \%)$. In the literature, there is debate over memory for searched locations (that remain visible). Cain and Mitroff (2103) argued that having selected targets disappear may free up resources (such as working memory) for other tasks. But note that this conclusion may be in direct contrast with the results of Thornton \& Horowitz (2008) who found that there was little effect of leaving distractors on the screen upon search performance. In any case: leaving the targets in place did not seem to have a large effect on performance, at least in this paradigm.

In Experiment 2 we addressed the question of whether the manipulation of not making items disappear may have affected strategies. This was not the case: in Experiment 2 the items disappeared once they had been tapped, yet performance was similar to what it was in experiment 1 . Furthermore, in Experiment 3 we tested whether the strong penalty for errors in experiments 1 and 2 had affected the results, by decreasing the penalty (withdrawing points rather than cancelling the trial 
and having observers start over), but found that again the foraging patterns were roughly identical.

\section{Further Considerations}

Another interesting finding from the perspective of the marginal value theorem in particular, is that even though the cost of leaving a patch and moving to a new one is low (the new patch is visible immediately after the observers press the button, and the cost is really only the few hundred milliseconds that it takes them to press the button that displays a new patch on the next screen refresh), observers stay for very long within the patch, even during conjunction foraging (the average collection rate is $>20$, even during conjunction foraging).

We should note that our task could be criticized for stacking the deck against MVT and OFT. One notable point is for example that the acquisition rates do not decrease as the foraging trials progress, as is assumed by the theory. This is certainly a valid consideration but from our perspective, such accounts cannot cherry-pick foraging paradigms - the account should apply to many different foraging scenarios. Our results may therefore set some boundary conditions regarding applications of concepts such as the MVT.

Overall regarding the question of whether the marginal value theorem and optimal foraging theories based in it can account for performance, we think that the most interesting question may not be whether the behavior accords well with a mathematical theorem such as the marginal value theorem. This may seem surprising in light of the emphasis that we have laid on the theorem. But in fact, some argue that optimal foraging theory is a "total waste of time" as a theoretical construct in biology, and that it "has no place in current evolutionary thought: its use is a throwback to the comfortable determinism of Divine Creation; to the endeavours of natural philosophers seeking to demonstrate the wisdom of the Creator" (Pierce \& Ollason, 1987). This rather colorful quote reflects the view that evolution would not optimize behavior in such a way and that behavior is determined by many variables that may be too random for such optimization to occur. We most certainly, do not wish to go as far as Pierce and Ollason do, yet a viable theoretical account of human visual foraging would need to take into account the relation between the stimulus characteristics and the task requirements. What matters in the end is what determines behavior. Choices to leave a patch are made differently for different tasks. For example, Wolfe (2013) found that MVT applied well to some straightforward foraging tasks but sometimes collection rates were lower than MVT would predict, and observers should have left the patch sooner, before collection rates dropped. Wolfe (2013, experiment 5) manipulated patch quality while in another experiment participants did not know whether the berries were good or bad (Wolfe,
2013, experiment 6). In both these cases patch leaving diverged from the predictions of the marginal value theorem. The results indicated that observers seemed to use another quitting rule than the instantaneous rate of return, and the cost of moving to a new patch did not straightforwardly affect when observers moved to a new patch. In Wolfe et al, 2018, different foraging targets had different value and their prevalence was varied, and in both these cases, performance deviated from the MVT predictions and was strongly modulated by the value of each item.

We should also note that selection times when observers select the same target as during their last selection were virtually identical for feature and conjunction foraging. It is a wellknown finding in the visual search literature that search times are far higher for conjunction than feature search so this similarity is somewhat surprising. We should note, however that switching between target types on consecutive selections is much slower for conjunction than feature foraging. Note also that there was remarkable similarity in the number of targets that observers collected before moving to the next patch between conditions although the conjunction foraging condition is much harder overall, but observers seem to compensate for this by changing their foraging strategy. We speculate that their repeated selections of the same target type are facilitated by priming of attention shifts (Brascamp et al., 2011; Shurygina et al., 2019; see Kristjánsson \& Ásgeirsson, 2019).

A reviewer noted that the foraging patterns that we see may reflect "planning ahead" by observers. In essence, this view entails that observers have already attentionally selected the items that they subsequently tap, but the pace of motor selection limits their observed selection rates. We believe that it is quite obvious that planning ahead and motor limitations are involved in any foraging task, but we also think that this cannot easily explain all aspects of the performance patterns we see. Recent data from our lab where foraging items move around throughout each trial (presumably affecting planning ahead) supports this, since in spite of the items moving around, performance was similar to what we have seen in previous experiments with static displays.

One final point that we wish to make is that in some ways our results may agree with the marginal value theorem. As figure 1 shows, according to MVT, participants should stay longer in a patch when the overall acquisition rate across all patches is lower, Consistent with this, the runs are longer for conjunction than feature foraging, and the acquisition rate is lower for conjunction foraging in experiments and 1 and 2 . Second, a possible interesting interpretation of the results is that they reveal patch foraging between categories within the same patch. The fact that participants switch more often during feature than conjunction foraging, and the larger switch costs for conjunction foraging could be considered another form of patch switching. We believe that this certainly warrants further study in future experiments. 


\section{Conclusions}

We tested foraging using Anne Treisman's classic feature versus conjunction manipulation. We designed our iPad foraging task to involve more dynamic assessment of attentional orienting than visual search studies provide while still allowing good control of independent variables. The aim was to assess whether foraging would follow the predictions of optimal foraging theories that incorporate the marginal value theorem. We found that performance on the foraging task does not follow the predictions of the marginal value theorem, with the most notable deviation being that observers foraged for longer within each patch than expected, although the fact that acquisition rates did not decrease in our study may complicate interpretations with regard to OFT and the MVT. But overall, we agree with Wolfe (2013) who suggested that while many aspects of foraging performance may be shared with our animal relatives, other demands of daily life may require adjustment of simple rules. Many other variables determine performance in such a dynamic task that a simple mathematical equation may not fully capture.

Acknowledgements We thank Thomas Hills, Jeremy Wolfe and two anonymous reviewers for invaluable comments on a previous draft. The research was supported by Icelandic Research Fund (IRF \#152427) and the Research fund of the University of Iceland

\section{References}

Bartumeus, F., \& Catalán, J. (2009). Optimal search behavior and classic foraging theory. Journal of Physics A: Mathematical and Theoretical, 42(43), 434002.

Baum, W. M. (1987). Random and systematic foraging, experimental studies of depletion, and schedules of reinforcement. In Foraging behavior (pp. 587-607). Springer, Boston, MA.

Biggs, A. T., \& Mitroff, S. R. (2015). Improving the efficacy of security screening tasks: A review of visual search challenges and ways to mitigate their adverse effects. Applied Cognitive Psychology, 29(1), 142-148.

Bond AB (1982) The bead game: Response strategies in free assortment. Human Factors, 24, 101-110.

Bond AB (2007) The evolution of color polymorphism: Crypticity, searching images, and apostatic selection. Annual Review Ecological Evolution, 38, 489-514.

Brascamp, J.W., Blake, R. \& Kristjánsson, Á. (2011). Deciding where to attend: priming of pop-out drives target selection. Journal of Experimental Psychology: Human Perception \& Performance, 37, 1700-1707.

Cain, M. S., \& Mitroff, S. R. (2013). Memory for found targets interferes with subsequent performance in multiple-target visual search. Journal of Experimental Psychology: Human Perception and Performance, 39(5), 1398.

Cain, M. S., Vul, E., Clark, K., \& Mitroff, S. R. (2012). A Bayesian optimal foraging model of human visual search. Psychological Science, 23(9), 1047-1054.

Cain, M. S., Boettcher, S. E., \& Wolfe, J. M. (2014). When Does the Aardvark Move to the Next Anthill? Foraging search with moving targets. Journal of Vision, 14(10), 919-919.
Charnov, E. L. (1976). Optimal foraging, the marginal value theorem. Theoretical Population Biology, 9(2), 129-136.

Dawkins, M. (1971). Shifts of 'attention' in chicks during feeding. Animal Behavior, 19, 575-582.

Dukas, R. (2002). Behavioural and ecological consequences of limited attention. Philosophical Transactions of the Royal Society, B: Biological Sciences, 357(1427), 1539-1547.

Dukas, R., \& Ellner, S. (1993). Information processing and prey detection. Ecology, 74(5), 1337-1346.

Dukas, R., \& Kamil, A. C. (2001). Limited attention: The constraint underlying search image. Behavioral Ecology, 12(2), 192-199.

Duncan, J., \& Humphreys, G. W. (1989). Visual search and stimulus similarity. Psychological Review, 96(3), 433.

Eckstein, M. P. (1998). The lower visual search efficiency for conjunctions is due to noise and not serial attentional processing. Psychological Science, 9(2), 111-118.

Egeth, H., Jonides, J., \& Wall, S. (1972). Parallel processing of multielement displays. Cognitive Psychology, 3(4), 674-698.

Hills TT (2006) Animal foraging and the evolution of goal-directed cognition. Cognitive Science, 30, 3-41.

Hills TT, Kalff C, Wiener JM (2013) Adaptive Lévy processes and arearestricted search in human foraging. PLoS ONE 8(4), e60488

Kamil AC, Bond AB (2006) Selective attention, priming, and foraging behaviour. In: Zentall TR, Wasserman E, editors. Comparative cognition: experimental explorations of animal intelligence (pp. 106126). Oxford: Oxford University Press

Kamil, A. C., Yoerg, S. I., \& Clements, K. C. (1988). Rules to leave by: Patch departure in foraging blue jays. Animal Behaviour, 36(3), 843853.

Jóhannesson, Ó. I., Thornton, I. M., Smith, I. J., Chetverikov, A., \& Kristjánsson, Á. (2016). Visual foraging with fingers and eye gaze. i- Perception, 7(2), 2041669516637279.

Jóhannesson, Ó.I., Kristjánsson, Á. \& Thornton, I.M. (2017). Are foraging patterns in humans related to working memory and inhibitory control? Japanese Psychological Research, 59, 152-166.

Kristjánsson, Á. (2015). Reconsidering visual search. i-Perception, 6(6), 2041669515614670.

Kristjánsson, Á. \& Ásgeirsson, Á.G. (2019). Attentional Priming: Recent insights and current controversies. In the press in Current Opinion in Psychology, 29, 71-75.

Kristjánsson, Á \& Egeth, H. (2019). How feature integration theory integrated cognitive psychology, neurophysiology, and psychophysics. In the press in Attention, Perception \& Psychophysics, 29, 1-7. https://doi.org/10.3758/s13414-019-01803-7.

Kristjánsson, T., Draschkow, D., Pálsson, Á., Haraldsson, D., Jónsson, P.O. \& Kristjánsson, Á. (2019). Visual foraging in the "real world", Presentation at the European Conference on Visual Perception.

Kristjánsson, Á., Jóhannesson, Ó.I. \& Thornton, I.M. (2014). Common Attentional Constraints in Visual Foraging. PLOS One 9 (6), e100752.

Kristjánsson, T. \& Kristjánsson, Á (2018). Foraging through multiple target categories reveals the flexibility of visual working memory. Acta Psychologica, 183, 108-115.

Kristjánsson, Á., Ólafsdóttir, I.M. \& Kristjánsson, T. (in press). Visual foraging tasks provide new insights into the orienting of visual attention: Methodological considerations. Springer Neuromethods.

Kristjánsson, T., Thornton, I.M., \& Kristjánsson, Á. (2018). Time limits during visual foraging reveal flexible working memory templates. Journal of Experimental Psychology: Human Perception and Performance, 44(6), 827-835.

Kristjánsson, T., Thornton, I.M., Chetverikov, A. \& Kristjánsson, Á. (2020). Dynamics of visual attention revealed in foraging tasks. Cognition, 194, 104032

Langley, C.M., Riley, D.A., Bond, A.B,. Goel, N. (1995) Visual search for natural grains in pigeons: search images and selective attention. 
Journal of Experimental Psychology: Animal Behavior, 22, 139151.

Mook, J. H., Mook, L. J., \& Heikens, H. S. (1960). Further evidence for the role of "searching images" in the hunting behaviour of titmice. Archives Neerlandaises de Zoologie, 13(3), 448-465.

Neisser, U. (1963). Decision-time without reaction-time: Experiments in visual scanning. The American Journal of Psychology, 76(3), 376385 .

Neisser, U. (1964). Visual search. Scientific American, 210(6), 94-103.

Ólafsdóttir, I.M., Kristjánsson, T., Gestsdóttir, S., Jóhannesson, Ó.I., Kristjánsson, Á. (2016). Understanding visual attention in childhood: Insights from a new visual foraging task. Cognitive Research: Principles and Implications.

Palmer, J. (1994). Set-size effects in visual search: The effect of attention is independent of the stimulus for simple tasks. Vision Research, 34(13), 1703-1721.

Pierce, G. J. \& Ollason, J. G. (1987). Eight reasons why optimal foraging theory is a complete waste of time. Oikos, 49, 111-118.

Pyke, G. H., Pulliam, H. R., \& Charnov, E. L. (1977). Optimal foraging: A selective review of theory and tests. The Quarterly Review of Biology, 52(2), 137-154.

Shurygina, O., Kristjánsson, Á., Tudge, L. \& Chetverikov, A. (2019). Expectations and perceptual priming in a visual search task: Evidence from eye movements and behavior. In the press in the Journal of Experimental Psychology: Human perception and performance, 45(4), 489.

Soce, A. A., Cain, M. S., \& Wolfe, J. M. (2016). Fitting two target templates into the focus of attention in a hybrid foraging task. Poster presented at the annual meeting of the Vision Sciences Society.

Staddon, J. E. R., \& Gendron, R. P. (1983). Optimal detection of cryptic prey may lead to predator switching. The American Naturalist, $122(6), 843-848$
Stephens, D. W., Brown, J. S., \& Ydenberg, R. C. (2007). Foraging: Behavior and Ecology. Chicago: The University of Chicago Press.

Thornton, I. M., \& Horowitz, T. S. (2004). The multi-item localization (MILO) task: Measuring the spatiotemporal context of vision for action. Perception \& Psychophysics, 66(1), 38-50.

Tinbergen, L. (1960). The natural control of insects in pinewoods I. Factors influencing the intensity of predation by songbirds. Archives Néerlandaises de Zoologie, 13, 265-336.

Treisman, A. (1977). Focused attention in the perception and retrieval of multidimensional stimuli. Perception \& Psychophysics, 22(1), 1-11.

Treisman, A. (1988). Features and objects: The fourteenth Bartlett memorial lecture. The Quarterly Journal of Experimental Psychology, 40(2), 201-237.

Treisman, A. M., \& Gelade, G. (1980). A feature-integration theory of attention. Cognitive Psychology, 12(1), 97-136.

Treisman, A., Sykes, M., \& Gelade, G. (1977). Selective attention and stimulus integration. Attention and performance VI, 333.

Wolfe, J. M. (1994). Guided search 2.0 a revised model of visual search. Psychonomic Bulletin \& Review, 1(2), 202-238.

Wolfe, J. M. (2013). When is it time to move to the next raspberry bush? Foraging rules in human visual search. Journal of Vision, 13(3), 1010.

Woods, A. J., Göksun, T., Chatterjee, A., Zelonis, S., Mehta, A., \& Smith, S. E. (2013). The development of organized visual search. Acta Psychologica, 143(2), 191-199.

Zhang, J., Gong, X., Fougnie, D. \& Wolfe, J.M. (2017). How humans react to changing rewards during visual foraging. Attention, Perception, \& Psychophysics 79 (8), 2299-2309.

Publisher's note Springer Nature remains neutral with regard to jurisdictional claims in published maps and institutional affiliations. 